BASRENDER

Trascender, Contabilidad y Gestión Núm. 10 (enero - abril del 2019).

ISSN: 2448-6388. Universidad de Sonora. Departamento de Contabilidad.

Reserva de Derechos 04-2015-04172070800-203.

\title{
Importancia de la aplicación de los principios fundamentales de auditoría del sector público en las Entidades de Fiscalización Superior Importance of the application of the fundamental principles of public sector audit at Higher Audit Entities
}

Martin Gutiérrez Piñeira ${ }^{1}$

\section{Resumen}

La aplicación de los principios fundamentales de auditoría del sector público en las entidades de fiscalización superior es de gran importancia tanto para los auditores de las entidades de fiscalización superior, las entidades fiscalizadas y terceros interesados, por lo que la Organización Internacional de Entidades Fiscalizadoras Superiores (INTOSAI) muestra la relevancia de apegarse a la normativa, y su fácil adopción, adaptación y aplicación. Para que las entidades de fiscalización superior realicen sus funciones de manera eficaz y eficiente y así señalar las desviaciones normativas que las entidades fiscalizadas infringen en el ejercicio del gasto público, no solo en la operación, sino en la actividad total de la administración, de tal forma que el sujeto fiscalizado pueda adoptar medidas correctivas en cada caso y así se logre evitar reincidencias.

Para la realización de este artículo se utilizó la técnica de investigación documental la cual como dice el autor "La investigación documental consiste en un análisis de la información escrita sobre un determinado tema, con el propósito de establecer relaciones, diferencias, etapas, posturas o estado actual del conocimiento respecto al tema objeto de estudio. (Bernal, C.A. 2010 p.111). y de acuerdo con Casares Hernández, et al. (1995), la investigación documental depende fundamentalmente de la información que se obtiene o se consulta en documentos, entendiendo por éstos todo material al que se puede acudir como fuente de referencia, sin que se altere su naturaleza o sentido, los cuales aportan información o dan testimonio de una realidad o un acontecimiento (Bernal, C.A. 2010 p.111).

La Organización Internacional de Entidades Fiscalizadoras Superiores (INTOSAI), globalmente, y la Organización Latinoamericana y del Caribe de Entidades Fiscalizadoras Superiores (OLACEFS), regionalmente, son organismos internacionales por excelencia en materia de auditoría gubernamental, que hoy en día son un referente para que las entidades de fiscalización superior, con la debida consideración de sus mandatos y regímenes legales y contextos, puedan implementar normas que coadyuven al adecuado ejercicio de la labor de auditoría gubernamental.

El lema de la Organización Internacional de Entidades Fiscalizadoras Superiores (INTOSAI) "La experiencia mutua beneficia a todos", resume y justifica la relevancia de intervenir en iniciativas internacionales.

Palabras clave: entidades fiscalizadoras, auditoría, Normas Internacionales de las Entidades Fiscalizadoras Superiores (ISSAIs, por sus siglas en inglés).

\footnotetext{
${ }^{1}$ Martín Gutiérrez Piñeira. Contador Público. Auditor del Instituto Superior de Auditoría y Fiscalización de Sonora (ISAF). Correo: cpmgutierrez@hotmail.com
} 


\section{Abstract}

The application of the fundamental principles of auditing of the public sector in the entities of superior control is of great importance for both the auditors of the entities of superior control, the audited entities and interested third parties, for which the International Organization of Supreme Audit Institutions ( INTOSAI) shows the relevance of adhering to regulations, and their easy adoption, adaptation and application. So that the entities of superior control perform their functions in an efficient and efficient way and thus point out the normative deviations that the audited entities infringe in the exercise of the public expense, not only in the operation, but in the total activity of the administration, of such so that the taxpayer can take corrective measures in each case and thus avoid recidivism.

For the realization of this article we used the technique of documentary research which as the author says "The documentary research consists of an analysis of written information on a certain topic, with the purpose of establishing relationships, differences, stages, positions or status current knowledge about the subject under study. (Bernal, C.A. 2010: 111). and according to Casares Hernández, et al. (1995), documentary research

DOI: https://doi.org/10.36791/tcg.v10i0.59

JEL: M42 Auditoría.

Recibido: 14 de diciembre de 2018.

Aceptado: 26 de marzo de 2019. depends fundamentally on the information obtained or consulted in documents, understood as all material that can be used as a reference source, without altering its nature or meaning, which provide information or they bear witness to a reality or an event. (Bernal, C.A. 2010: 111).

The International Organization of Supreme Audit Institutions (INTOSAI), globally, and the Latin American and Caribbean Organization of Supreme Audit Institutions (OLACEFS), regionally, are international organizations par excellence in matters of government audit, which today are a reference for the entities of superior control, with the due consideration of their mandates and legal regimes and contexts, can implement norms that contribute to the proper exercise of the government audit work.

The motto of the International Organization of Supreme Audit Institutions (INTOSAI) Mutual experience benefits everyone", summarizes and justifies the relevance of intervening international initiatives.

Keywords: audit institutions, audit, International Standards of Supreme Audit Institutions (ISSAIs, for its acronym in English). 


\section{Introducción}

Las normas profesionales son esenciales para la credibilidad, calidad y profesionalismo de las auditorías del sector público. Las Normas Internacionales de las Entidades Fiscalizadoras Superiores (ISSAIs, por sus siglas en inglés) desarrolladas por la Organización Internacional de las Entidades Fiscalizadoras Superiores (INTOSAI, por sus siglas en inglés) tienen por objetivo promover una auditoría eficaz e independiente, así como apoyar a los miembros de la INTOSAI en el desarrollo de su propio enfoque profesional en conformidad con sus mandatos y con las leyes y reglamentos nacionales, La fiscalización del sector público, realizada por entidades de fiscalización superior es un factor importante en la gobernanza pública ya que tiene un impacto positivo en la confianza de la sociedad.

\section{Antecedentes}

En el Diario Oficial de la Federación el 18 de julio del 2016, Se expide la Ley General del Sistema Nacional Anticorrupción la cual es de observancia general en todo el territorio nacional, y coordinara a las autoridades de todos los órdenes de gobierno, en la prevención, detección y sanción de responsabilidades administrativas, de hechos de corrupción, así como en la fiscalización y control de recursos públicos, y es integra por un Comité Coordinador, Comité de Participación Ciudadana, Comité Rector del Sistema Nacional de Fiscalización $y$ Los Sistemas Locales, quienes concurrirán a través de sus representantes.

"La presente Ley es de orden público, de observancia general en todo el territorio nacional y tiene por objeto establecer las bases de coordinación entre la Federación, las entidades federativas, los municipios y las alcaldías de la Ciudad de México, para el funcionamiento del Sistema Nacional previsto en el artículo 113 de la Constitución Política de los Estados Unidos Mexicanos, para que las autoridades competentes prevengan, investiguen y sancionen las faltas administrativas y los hechos de corrupción” (Ley General del Sistema Nacional Anticorrupción Art. 1).

"El Sistema Nacional de Fiscalización tiene por objeto establecer acciones y mecanismos de coordinación entre los integrantes del mismo, en el ámbito de sus respectivas competencias, promoverán el intercambio de información, ideas $y$ experiencias encaminadas a avanzar en el desarrollo de la fiscalización de los recursos públicos. Son integrantes del Sistema Nacional de Fiscalización, la Auditoría Superior de la Federación, la Secretaría de la Función Pública, las entidades de fiscalización superiores locales y las secretarías o instancias homólogas encargadas del control interno en las entidades federativas”. (Ley General del Sistema Nacional Anticorrupción Art. 37).

Así mismo en el Diario Oficial de la Federación el 18 de julio del 2016 Se expide la Ley General de Responsabilidades Administrativas, la cual establece que habrá faltas administrativas graves y no graves de los servidores públicos y de particulares, así como las sanciones por sus actos u omisiones.

"La presente Ley es de orden público y de observancia general en toda la República, y tiene por objeto distribuir competencias entre los órdenes de gobierno para establecer las responsabilidades administrativas de los Servidores Públicos, sus obligaciones, las sanciones aplicables por los actos $u$ omisiones en que estos incurran y las que correspondan a los particulares vinculados con faltas administrativas graves, así como los procedimientos para su aplicación”. (Ley General de Responsabilidades Administrativas Art. 1). 
De igual forma en el Diario Oficial de la Federación el 18 de julio del 2016 se expide la Ley de Fiscalización y Rendición de Cuentas de la Federación, para reglamentar las leyes que regulen la organización y facultades de la Auditoría Superior de la Federación, así como coordinar y evaluar sus funciones conforme a los principios de legalidad, definitividad, imparcialidad y confiabilidad.

\section{“...La Auditoría Superior de la Federación} podrá fiscalizar las operaciones que involucren recursos públicos federales o participaciones federales a través de contrataciones, subsidios, transferencias, donativos, fideicomisos, fondos, mandatos, asociaciones público-privadas o cualquier otra figura jurídica y el otorgamiento de garantías sobre empréstitos de Estados y Municipios, entre otras operaciones...” (Ley de Fiscalización y Rendición de Cuentas de la Federación Art. 1)

El 14 de julio del 2008 se expide la ley 168, Ley de Fiscalización para el Estado de Sonora en 168; B. O. No. 4 SECCION II, y en el decreto No. 186; B. O. 14, sección II, se presenta la reforma de fecha 15 de febrero de 2018, la cual tiene la cual tiene por objeto “...regular la revisión, auditoría y fiscalización superior de los estados financieros $\mathrm{y}$ cuentas públicas estatal y municipal...” (Ley de Fiscalización Superior para el Estado de Sonora Art.1)

"En los procedimientos de fiscalización se utilizarán las Normas de Auditoría Generalmente Aceptadas, las Normas de Información Financiera emitidas por el Instituto Mexicano de Contadores Públicos, los Postulados Básicos de Contabilidad Gubernamental, Normas Profesionales del Sistema Nacional de Fiscalización, las mejores prácticas y toda aquella disposición normativa aplicable en la materia," (Ley de Fiscalización Superior para el Estado de Sonora Art.23 fraccII)

\section{Objetivo}

La aplicación de los principios fundamentales de auditoría del sector público en las entidades de fiscalización superior es de gran importancia tanto para los auditores de las entidades de fiscalización superior, las entidades fiscalizadas y terceros interesados, por lo que la Organización Internacional de Entidades Fiscalizadoras Superiores (INTOSAI) muestra la relevancia de apegarse a la normativa, y su fácil adopción, adaptación y aplicación. Para que las entidades de fiscalización superior realicen sus funciones de manera eficaz y eficiente y así señalar las desviaciones normativas que las entidades fiscalizadas infringen en el ejercicio del gasto público, no solo en la operación, sino en la actividad total de la administración, de tal forma que el sujeto fiscalizado pueda adoptar medidas correctivas en cada caso y así se logre evitar reincidencias.

\section{Metodología}

Para la realización de esta investigación se utilizó la técnica de investigación documental la cual como dice el autor "La investigación documental consiste en un análisis de la información escrita sobre un determinado tema, con el propósito de establecer relaciones, diferencias, etapas, posturas o estado actual del conocimiento respecto al tema objeto de estudio. (Bernal, C.A. 2010: 111). y de acuerdo con Casares Hernández, et al. (1995), la investigación documental depende fundamentalmente de la información que se obtiene o se consulta en documentos, entendiendo por éstos todo material al que se puede acudir como fuente de referencia, sin que se altere su naturaleza o sentido, los cuales aportan información o dan testimonio de una realidad o un acontecimiento (Bernal, C.A. 2010: 111).

\section{Desarrollo}

Hoy en día ningún país puede estar alejado de los acontecimientos mundiales y centrarse individualmente e interiormente a controlar su forma de ejercer el control 
en la gobernanza nacional, sin verse afectado por los factores externos, para lo cual deben contar con sistemas de fiscalización que se integren por entidades de fiscalización superior las cuales contribuyen a incrementar la transparencia, garantizar la rendición de cuentas, promover el buen desempeño y luchar contra la corrupción, mejorando así la buena gobernanza e impulsando la equidad, ejerciendo sus procedimientos de auditoría y fiscalización en la normatividad y buenas prácticas aplicables en la materia, apoyándose en organismos internacionales como la "INTOSAI que es un organismo autónomo, independiente y apolítico, una organización no gubernamental con un estatus especial con el Consejo Económico y Social de las Naciones Unidas (ECOSOC), ha sido fundada en el año 1953 a iniciativa del entonces presidente de la EFS de Cuba, Emilio Fernández Camus” (INTOSAI, 2018).

La Organización Internacional de Entidades Fiscalizadoras Superiores (INTOSAI) "proporciona un marco de trabajo institucionalizado a las Entidades Fiscalizadoras Superiores (EFS), con el fin de promover el desarrollo y la transferencia de conocimientos, la mejora de la auditoría del sector público en todo el mundo y el aumento de las capacidades profesionales, así como la presencia e influencia de sus miembros en sus respectivos países.

La INTOSAI se ocupa de las Normas Internacionales de Entidades Fiscalizadoras Superiores las también llamadas ISSAI, por sus siglas en inglés, son normas profesionales emitidas con la aprobación del Congreso de la INTOSAI" (Marco normativo de la INTOSAI 2018).

La aplicación de las ISSAI asegura que se instrumentan normas que han pasado por un proceso de revisión, evitando la arbitrariedad, y atendiendo las mejores prácticas (Véase Figura 1).

"Promoción de la eficiencia, la rendición de cuentas, la eficacia y la transparencia de la administración pública mediante el fortalecimiento de las entidades fiscalizadoras superiores" (Asamblea General de las Naciones Unidas, 2011). 
Figura 1. Aplicación de las ISSAI

\section{La aplicación de las ISSAI:}

Fuente: Elaboración propia.

La importancia de implementar adecuadamente las ISSAI, como se señala en la Declaración de Beijing en su punto 2, las entidades de fiscalización superior tienen, entre otras responsabilidades, la de contribuir con su labor auditora al fortalecimiento de las finanzas públicas. Para ello, las EFS "pueden ayudar a quienes son responsables de la toma de decisiones, mediante sus informes de auditoría, a salvaguardar la sostenibilidad a largo plazo y el cumplimiento de las políticas financieras, incrementando así la transparencia, informando acerca de las finanzas públicas y revelando al mismo tiempo sus riesgos sistémicos.”
La ejecución de las normas de la INTOSAI “...conlleva a beneficios muy significativos, debido a que son desarrolladas por expertos de alto nivel y experiencia tras la colaboración con organismos internacionales, cuyo reconocimiento garantiza la aplicabilidad y revisión periódica de los criterios de auditoría, asegurando una normativa actualizada y vanguardista..." (ICADEFIS $2^{\text {a }}$ edición, V.1, enero 2018: 5). (Véase Figura 2) 
Figura 2: El valor de la INTOSAI

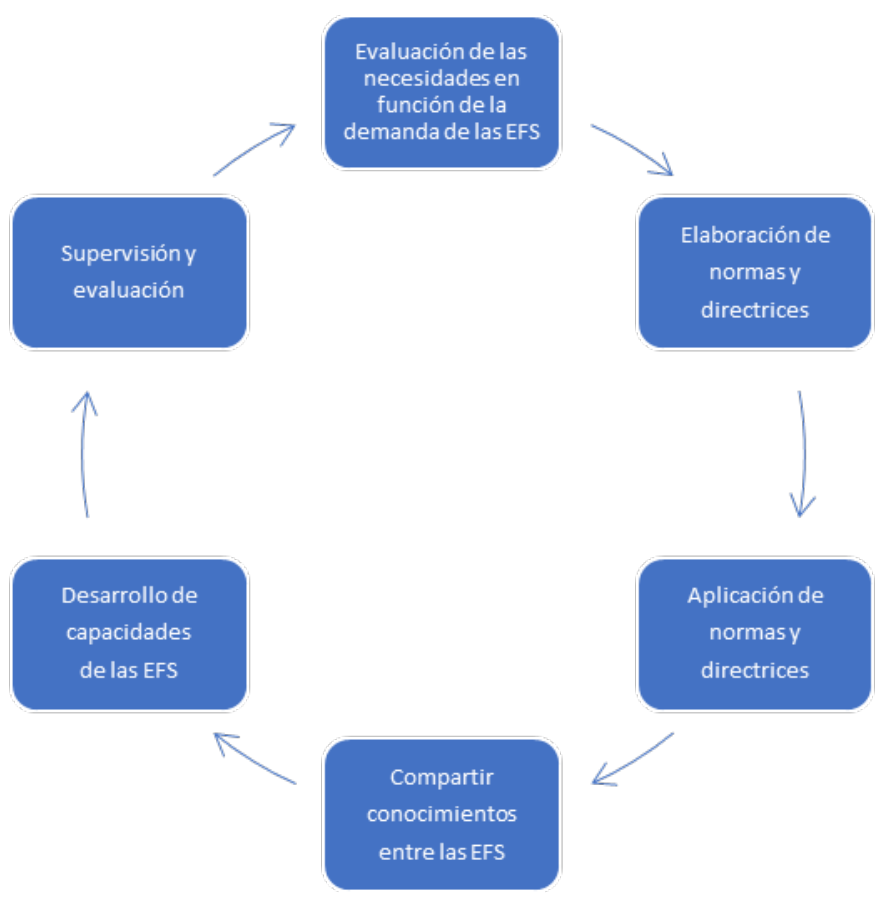

Fuente: Elaboración propia en base INTOSAI, 2018.

La estructura orgánica de la INTOSAI está conformada por el Congreso de la INTOSAI como máxima instancia, por el Comité Directivo del cual derivan la Iniciativa para el Desarrollo de la INTOSAI (IDI), la Revista Internacional de Auditoría Gubernamental (INTOSAI Journal), las Organizaciones Regionales y la Secretaría General” (ICADEFIS 2a edición, V.1, enero 2018 p.8), la INTOSAI reconoce a las Organizaciones Regionales como entidades relacionadas autónomas creadas con el fin de fomentar, sobre una base regional, la cooperación profesional y técnica de sus miembros. Entre sus organizaciones regionales se encuentra la Organización Latinoamericana y del Caribe de Entidades Fiscalizadoras Superiores (OLACEFS) un organismo autónomo, independiente, apolítico y de carácter permanente que tiene por objetivo fomentar el desarrollo y el perfeccionamiento de sus miembros a través de las siguientes acciones, la capacitación y especialización, la investigación científica y el desarrollo de estudios técnicos, la asesoría y asistencia técnica, la información y coordinación vinculada con las actividades de la Organización y otras acciones que le permitan el cumplimiento de sus objetivos. Como organización regional de las Entidades de Fiscalización Superior (EFS) de Latinoamérica y del Caribe, México preside el periodo 2016 - 2018, a través de la Auditoría Superior de la Federación.

Existen diversas organizaciones que se han encargado de emitir normas internacionales para las actividades de auditoría y fiscalización, para promover las mejores prácticas para realizar estas tareas por medio de directrices y consejos que se constituyen como la normativa internacional en las que apoya su labor la Auditoría Superior de la Federación. 
Las principales organizaciones que han emitido normativa al respecto son las siguientes:

\section{Federación Internacional de Contadores (IFAC por sus siglas en inglés)}

La Federación Internacional de Contadores (IFAC, por sus siglas en inglés), fue fundada en octubre de 1977 en Munich, Alemania, en el $11^{\circ}$. Congreso Mundial de Contadores, estableciéndose para fortalecer la profesión contable en el interés público mundial.

\section{Instituto de Auditores Internos (IIA por sus siglas en inglés)}

El IIA, fundado en 1941 es una asociación de profesionales especialistas en auditoría interna,

administración de riesgos, gobierno corporativo, control interno, auditoría a tecnología de información, educación y seguridad y auditoría gubernamental. Cuenta con más de 185,000 miembros de todo el mundo.

\section{Consejo de Normas Internacionales de Contabilidad (IASB por sus siglas en inglés)}

El Consejo de Normas Internacionales de Contabilidad (International Accounting Standards Board) es un organismo independiente del sector privado que desarrolla y aprueba las Normas Internacionales de Información Financiera. (ICADEFIS $2^{\mathrm{a}}$ edición, V.1, enero 2018: 8).

La INTOSAI se ocupa de las Normas Internacionales de las Entidades Fiscalizadoras Superiores. Las también llamadas ISSAIs, por su acrónimo en inglés, son normas profesionales emitidas con la aprobación del Congreso de la INTOSAI, su máxima autoridad. Éstas contienen los prerrequisitos básicos para el funcionamiento adecuado de los organismos auditores y los principios fundamentales de auditoría a entidades públicas.
Incluyen, además, recomendaciones sobre los requisitos previos legales, de organización y de índole profesional, así como sobre la conducta de la auditoría, además de ejemplos o descripciones de mejores prácticas.

\section{Normas Internacionales de las Entidades Fiscalizadoras Superiores (ISSAIs)}

Las ISSAIs tienen el objetivo de salvaguardar independencia y efectividad de la actividad auditora, y apoyar a los miembros de la INTOSAI en el desarrollo de su propio enfoque profesional en función de su mandato. La primera serie completa de ISSAIs fue puesta en marcha en el XX Congreso de Johannesburgo, Sudáfrica, en 2010. A través de la Declaración de Sudáfrica, la INTOSAI ha convocado a sus miembros a utilizar el marco de trabajo ISSAI como marco de referencia común para la auditoría del sector público, medir su propio desempeño y lineamientos de auditoría mediante el uso de ISSAIs, e implementar las ISSAIs de acuerdo con su mandato, legislación nacional y regulaciones.

Las ISSAIs forman una jerarquía de pronunciamientos oficiales con cuatro niveles:

\section{Nivel 1 - Principios Fundamentales (ISSAI 1)}

El nivel 1 del marco de trabajo ISSAI contiene los principios fundamentales de la INTOSAI. La ISSAI 1, Declaración de Lima de 1977, hace un llamado al establecimiento de Entidades Fiscalizadoras Superiores efectivas y provee lineamientos en preceptos de auditoría.

\section{Nivel 2 - Prerrequisitos para el Funcionamiento de las Entidades Fiscalizadoras Superiores (ISSAIs10- 99)}

Los prerrequisitos para el Funcionamiento de las Entidades Fiscalizadoras Superiores contienen los 
pronunciamientos de la INTOSAI sobre las condiciones previas necesarias para el adecuado funcionamiento y conducción profesional de las Entidades Fiscalizadoras Superiores. Éstos incluyen principios y lineamientos sobre independencia, transparencia y rendición de cuentas, ética y control de calidad. Los prerrequisitos pueden referirse al mandato institucional y legislaciones nuevas, así como a los procedimientos establecidos y prácticas diarias de la organización y su personal.

Mediante la emisión de pronunciamientos de estos requisitos aceptados de manera general, la INTOSAI tiene el objetivo de proponer principios sólidos para el funcionamiento adecuado del sector público a nivel internacional.

\section{Nivel 3 - Principios Fundamentales de Auditoría (ISSAIs 100-999)}

Los Principios Fundamentales de Auditoría expresan la esencia de la auditoría del sector público. Contienen los pronunciamientos comunes hechos por miembros de la INTOSAI sobre los principios profesionales, reconocidos de manera general, que sustentan la práctica de auditorías efectivas e independientes a las entidades del sector público. Los principios son diseñados y elaborados sobre los principios fundamentales del ISSAI 1 "La Declaración de Lima” y proporcionan un marco de referencia internacional acreditado, que define la auditoría del sector público en sus formas contemporáneas. El propósito es avanzar y salvaguardar las buenas prácticas de auditoría, apoyar el desarrollo continuo de entidades fiscalizadoras superiores efectivas $\mathrm{y}$, proveer un fundamento profesional para la cooperación internacional dentro de la INTOSAI y sus organizaciones regionales. Los principios deben de preocuparse de la base del mandato individual y estrategias de la entidad fiscalizadora superior, y aplicada como sea relevante y apropiado para el trabajo de auditoría, considerando los prerrequisitos de independencia, transparencia y rendición de cuentas, ética y control de calidad.

Nivel 4 - Lineamientos de Auditoría (ISSAIs 10005999)

Los Lineamientos de Auditoría traducen los principios fundamentales de auditoría en lineamientos más específicos, detallados y operativos que pueden ser utilizados de manera cotidiana en la conducción de tareas de auditoría. El propósito de los lineamientos es el de proporcionar una base para las normas y manuales de auditoría del sector público, que puedan ser utilizados individualmente por los miembros de la INTOSAI.

Cada lineamiento tiene un alcance en su aplicación y puede ser adoptado totalmente o adaptado, según sea necesario, para reflejar las circunstancias individuales de cada jurisdicción. Dichas circunstancias pueden incluir mandatos legales, estrategias y capacidades futuras de la entidad fiscalizadora superior, así como el propósito y carácter específicos de las tareas de auditoría. Algunos de los lineamientos del nivel 4 incluyen requerimientos especiales referentes a la autoridad. Los Lineamientos Generales de Auditoría (ISSAIs 1000-4999) contienen los requerimientos recomendados sobre auditoría financiera, de desempeño y de cumplimiento y, proveen lineamientos adicionales al auditor. Son desarrollados y actualizados continuamente por subcomités especializados que definen la mejor práctica internacional reconocida en su momento y dentro de su alcance general de aplicación. Los Lineamientos sobre temas específicos (ISSAI 5000-5999) contienen lineamientos complementarios sobre temas específicos de auditoría u otras cuestiones importantes que pudiesen requerir atención especial de las entidades fiscalizadoras 
superiores. Estos lineamientos expresan las lecciones clave resultantes del intercambio de conocimientos y buenas prácticas entre los expertos de la INTOSAI (2018).

En el nivel tres se encuentran los Principios Fundamentales de Auditoría los cuales expresan la esencia de la auditoría del sector público, se presentan a continuación: ISSAI 100 - Principios Fundamentales de Auditoría del Sector Público, ISSAI 200 - Principios Fundamentales de la Auditoría Financiera, ISSAI 300 Principios Fundamentales de la Auditoría de Desempeño, ISSAI 400 - Principios Fundamentales de la Auditoría de Cumplimiento. (Las Normas Internacionales de las Entidades Fiscalizadoras Superiores (ISSAIs) son emitidas por la Organización Internacional de Entidades Fiscalizadoras Superiores (INTOSAI).

\section{ISSAI 100 - Principios Fundamentales de Auditoría del Sector Público}

Establece los principios fundamentales que se aplican a toda labor de auditoría del sector público, independientemente de su forma o contexto, Los Principios Fundamentales de Auditoría constituyen la parte medular de las Directrices Generales de Auditoría del nivel 4 del marco normativo de las ISSAI. Estos principios pueden utilizarse para establecer normas autoritativas en tres formas:

- Como base para que las EFS puedan desarrollar normas.

- Como base para la adopción de normas nacionales congruentes.

- Como base para la adopción de las Directrices Generales de Auditoría como normas.
Las EFS deben declarar las normas que van a aplicar al realizar auditorías; dicha declaración deberá estar disponible para los usuarios de los informes de las EFS. Cuando las normas estén fundamentadas en diversas fuentes en conjunto, esto también deberá declararse. Se espera que las EFS incluyan dichas declaraciones como parte de sus informes de auditoría; sin embargo, también podrán utilizar medios de comunicación más generales. Una EFS ejercerá su función de auditoría del sector público dentro de un arreglo constitucional específico y en virtud de su cargo y mandato, lo que le garantiza suficiente independencia y poder de discreción para llevar a cabo sus deberes. El mandato de una EFS puede definir sus responsabilidades generales en el campo de la auditoría del sector público, y proporcionar prescripciones adicionales relativas a las auditorías y otros trabajos a realizar. El entorno de la auditoría del sector público es aquel en el que los gobiernos y otras entidades del sector público se responsabilizan sobre el uso de recursos provenientes de la tributación y otras fuentes, para la prestación de servicios a los ciudadanos y otros beneficiarios.

Estas entidades deben rendir cuentas de su gestión y desempeño, así como del uso de los recursos, tanto a quienes se los proporcionan como a quienes dependen de los servicios prestados utilizando tales recursos, incluyendo a los ciudadanos.

La auditoría del sector público ayuda a crear las condiciones adecuadas y a fortalecer la expectativa de que las entidades del sector y los servidores públicos desempeñarán sus funciones de manera eficaz, eficiente y ética, y de acuerdo con las leyes y reglamentos aplicables. En general, la auditoría del sector público puede describirse como un proceso sistemático en el que de manera objetiva se obtiene y se evalúa la evidencia 
para determinar si la información o las condiciones reales están de acuerdo con los criterios establecidos. La auditoría del sector público es esencial, ya que proporciona, a los órganos legislativos y de supervisión, a los encargados de la gobernanza y al público en general, información y evaluaciones independientes y objetivas concernientes a la administración y el desempeño de las políticas, programas u operaciones gubernamentales.

\section{ISSAI 200 - Principios Fundamentales de la} Auditoría Financiera

Ha sido desarrollada para abordar los aspectos clave relacionados con una auditoría de estados financieros en el sector público, La auditoría financiera se enfoca en determinar si la información financiera de una entidad se presenta de conformidad con la emisión de información financiera y el marco regulatorio aplicable. El alcance de las auditorías financieras en el sector público puede estar definido por el mandato de las EFS como una serie de objetivos de auditoría adicionales a los objetivos de una auditoría de estados financieros preparados de conformidad con un marco de emisión de información financiera. El propósito de una auditoría de estados financieros es aumentar el grado de confianza de los posibles usuarios en dichos estados financieros. Esto se logra a través de él dictamen emitido por el auditor sobre si los estados financieros han sido preparados, en todos los aspectos importantes, de acuerdo con el marco de referencia para la emisión de información financiera

Los Principios Fundamentales de Auditoría Financiera son aplicables a todas las auditorías de estados financieros del sector público, ya sea que se trate de la totalidad del gobierno, partes del gobierno o entidades individuales. La referencia a la ISSAI 200 en los informes sólo debe hacerse si las normas de auditoría se han desarrollado o adoptado para cumplir totalmente con todos los principios relevantes de la ISSAI 200.
Se considera que un principio es relevante cuando trata acerca del tipo de auditoría o combinaciones de distintos tipos de auditoría, y las circunstancias o procedimientos son aplicables. Los principios no están de ninguna manera por encima de las leyes, reglamentos o mandatos nacionales.

\section{ISSAI 300 - Principios Fundamentales de la Auditoría de Desempeño}

La auditoría de desempeño es una revisión independiente, objetiva y confiable sobre si las acciones, sistemas, operaciones, programas, actividades u organizaciones del gobierno operan de acuerdo con los principios de economía, eficiencia y eficacia, y sobre si existen áreas de mejora. La auditoría de desempeño busca brindar nueva información, análisis o perspectivas, a menudo incluyen un análisis de las condiciones que son necesarias para garantizar que los principios de economía, eficiencia y eficacia puedan mantenerse. Estas condiciones pueden incluir prácticas y procedimientos de la buena administración para garantizar la entrega correcta y oportuna de los servicios, cuando corresponda, el impacto del marco regulatorio o institucional sobre el desempeño de la entidad auditada también debe ser tomado en consideración, La auditoría de desempeño promueve la rendición de cuentas al asistir a los responsables de la gobernanza y de las tareas de supervisión para mejorar el desempeño.

Lo logra evaluando si las decisiones de la legislatura o del ejecutivo son preparadas e implementadas eficiente y eficazmente, y si los contribuyentes o ciudadanos han recibido el justo valor por su dinero, ello no cuestiona las intenciones y decisiones del legislativo, pero examina si deficiencias en las leyes y reglamentos o su forma de implementación han evitado la consecución de los objetivos determinados. 
La auditoría de desempeño se enfoca en áreas en donde pueda añadir valor a los ciudadanos, $\mathrm{y}$ en las cuales tenga el mayor potencial para la mejora. Proporciona incentivos constructivos para que las partes responsables tomen las medidas apropiadas. La auditoría de desempeño promueve la transparencia al ofrecer al parlamento / congreso, a los contribuyentes y otras fuentes de financiamiento, aquellos grupos objetivo de las políticas gubernamentales y a los medios de comunicación, una visión de la administración y los resultados de las diferentes actividades del gobierno. Con ello contribuye de manera directa al brindar información útil al ciudadano mientras sirve como base para el aprendizaje y las mejoras. En la auditoría de desempeño, las EFS tienen libertad de decidir, dentro de su mandato, sobre qué, cuándo y cómo auditar, y no se les debería limitar la publicación de sus hallazgos.

$\mathrm{Al}$ igual que en todas las auditorías, los usuarios de los informes de las auditorías de desempeño desean confiar en la información que utilizan para tomar decisiones. Por lo tanto, esperan informes confiables que establezcan la posición (basada en la evidencia) de las EFS respecto al tema evaluado. En consecuencia, los auditores de desempeño deben, en todos los casos, proporcionar hallazgos basados en evidencia suficiente y apropiada, y gestionar activamente el riesgo de informes inapropiados. Sin embargo, normalmente no se espera que los auditores de desempeño emitan un dictamen general, comparable al dictamen de estados financieros, en el alcance de la economía, eficiencia y eficacia por parte de la entidad auditada. El objetivo de la auditoría determina el enfoque y diseño del trabajo. Podría ser simplemente para describir la situación.

Sin embargo, los objetivos normativos de la auditoría (¿las cosas son como deberían ser?) y los objetivos de auditoría analíticos (¿por qué las cosas no son como deberían ser?) son más propensos a agregar valor.
En todos los casos, los auditores deben considerar a qué se refiere la auditoría, qué organizaciones y organismos están involucrados y para quién serían podrían ser relevantes las recomendaciones finales. Los objetivos de la auditoría bien definidos se refieren a una sola entidad o un grupo identificable de acciones, sistemas, operaciones, programas, actividades u organizaciones gubernamentales.

\section{ISSAI 400 - Principios Fundamentales de la Auditoría de Cumplimiento}

El propósito de las auditorías de cumplimiento es proporcionar un grupo integral de los principios, normas y directrices para llevar a cabo auditorías de cumplimiento de un tema, tanto cualitativo como cuantitativo, el cual varía ampliamente en términos de su alcance y puede abordarse a través de diferentes enfoques de auditoría y formatos de informes. Las EFS sólo deben hacer referencia a los Principios Fundamentales de la Auditoría de Cumplimiento en los informes de auditoría, ya sea en el Informe del Auditor o en otros formatos de informes; siempre y cuando las normas que hayan desarrollado o adoptado cumplan cabalmente con todos los principios relevantes de la ISSAI 400. De ninguna manera, los principios están por encima las leyes, reglamentos o mandatos nacionales.

La auditoría de cumplimiento es una evaluación independiente para determinar si un asunto cumple con las autoridades aplicables identificadas como criterios. Las auditorías de cumplimiento se llevan a cabo evaluando si las actividades, operaciones financieras e información cumplen, en todos los aspectos significativos, con las autoridades que rigen a la entidad auditada. El objetivo de la auditoría de cumplimiento del sector público es, por lo tanto, permitir a la EFS evaluar si las actividades de las entidades públicas cumplen con las autoridades que las rigen. 
Esto involucra presentar informes sobre el grado en que la entidad auditada cumple con los criterios establecidos. Los informes pueden variar entre opiniones breves estandarizadas y diferentes formatos de conclusiones, presentados de manera extensa o breve. La auditoría de cumplimiento puede tratar sobre la regularidad (cumplimiento con los criterios formales, tales como leyes, regulaciones y convenios relevantes) o con la decencia (observancia de los principios generales que rigen una sana administración financiera y el comportamiento de los funcionarios públicos). Mientras que la regularidad es el enfoque principal de la auditoría de cumplimiento, también la decencia puede ser un asunto pertinente dado el contexto del sector público, en el que existen ciertas expectativas relacionadas con la administración financiera y el comportamiento de los funcionarios públicos. Dependiendo del mandato de la EFS, el alcance de la auditoría puede, por lo tanto, incluir aspectos sobre decencia, La auditoría de cumplimiento también puede también llevar a que las EFS, con poderes jurisdiccionales, emitan juicios y sanciones a aquellos responsables de la administración de los fondos públicos. Algunas EFS están obligadas a remitir, a las autoridades judiciales, los datos que puedan ser sujetos a persecución penal. En este contexto, el objetivo de la auditoría de cumplimiento puede ampliarse, y el auditor debe tener en cuenta los requisitos relevantes específicos al diseñar la estrategia o planeación de la auditoría y durante todo el proceso de esta. La auditoría de cumplimiento puede abarcar una amplia variedad de asuntos y puede realizarse para proporcionar una seguridad razonable o limitada, utilizando diferentes tipos de criterios, procedimientos de recopilación de evidencia y formatos de informes. Las auditorías de cumplimiento pueden ser compromisos de atestiguamiento o de elaboración de informes directos, o ambos a la vez. El informe de auditoría puede ser extenso o breve, y las conclusiones pueden expresarse de diferentes maneras: como un dictamen claro escrito sobre el cumplimiento o como una respuesta más elaborada a preguntas específicas de la auditoría.

\section{Conclusión}

La Organización Internacional de Entidades Fiscalizadoras Superiores (INTOSAI), globalmente, y la Organización Latinoamericana y del Caribe de Entidades Fiscalizadoras Superiores (OLACEFS), regionalmente, son organismos internacionales por excelencia en materia de auditoría gubernamental, que hoy en día son un referente para que las entidades de fiscalización superior, con la debida consideración de sus mandatos y regímenes legales y contextos, puedan implementar normas que coadyuven al adecuado ejercicio de la labor de auditoría gubernamental.

El lema de la Organización Internacional de Entidades Fiscalizadoras Superiores (INTOSAI) "La experiencia mutua beneficia a todos", resume y justifica la relevancia de intervenir en iniciativas internacionales.

\section{Referencias:}

Office, N. A. (2013). Declaración de Beijing. 86(1).

INTOSAI Professional Standards Committee. (2018). Recuperado de https://www.intosai.org/focus-areas

Asamblea General de las Naciones Unidas. (2011). Asuntos administrativos y presupuestarios. Acta 2011A/66/209. (diciembre 2011). Recuperado de https://www.un.org/es/ga/second/index.shtml

DOF. (2016a). Ley de Fiscalización y Rendición de Cuentas de la Federación. Diario Oficial de la Federación.

DOF. (2016b). Ley General de Responsabilidades Administrativas. Diario Oficial de la Federación.

DOF. (2016c). Ley General del Sistema Nacional Anticorrupción. Diario Oficial de la Federación.

DOF. (2017). Constitución Política de los Estados Unidos Mexicanos. Diario Oficial de la Federación.

DOF. (2018). Ley de Fiscalización Superior para el Estado de Sonora. Diario Oficial de la Federación. 